\title{
Apontamentos para a atuação do Profissional de Educação Física na Atenção Básica à Saúde: um ensaio
}

\section{Notes to the work of the Physical Education Professional in Basic Health Care:} an essay

\section{AUTORES \\ Mathias Roberto $\operatorname{Loch}^{1}$ (D) \\ Douglas Fernando Dias ${ }^{1}$ (D) \\ Cassiano Ricardo Rech $^{2}$ (D) \\ 1 Programa de Pós-Graduação em Saúde Coletiva, Universidade Estadual de Londrina. Paraná, Brasil. \\ 2 Programa de Pós-Graduação em Educação Física, Universidade Federal de Santa Catarina. Santa \\ Catarina. Brasil.}

\section{CONTATO}

Mathias Roberto Loch

matbias@uel.br

Departamento de Educação Física - Centro de Educação Física e Esporte. Universidade

Estadual de Londrina. Rodovia Celso Garcia Cid PR 445 - Campus Universitário.

Londrina, Paraná, Brasil.

CEP: 86051-980.

DOI

$10.12820 /$ rbafs. $24 \mathrm{e} 0069$

\begin{abstract}
RESUMO
O objetivo deste ensaio foi apresentar uma proposta de síntese para a atuação do profissional de Educação Física (PEF) no contexto da Atenção Básica à Saúde (ABS) do Brasil. São apresentadas 15 itens, elaborados a partir de experiências vivenciadas no campo de atuação pelos autores, bem como na literatura. Posteriormente, a clareza e pertinência dos itens foram avaliadas por $33 \mathrm{PEF}$ de nove diferentes municípios brasileiros que atuavam ou tinham atuado anteriormente na ABS. Apesar de os itens apresentados não terem o objetivo de competir ou substituir as diretrizes do Ministério da Saúde ou das secretarias estaduais ou municipais, espera-se que possam ser úteis para os PEF que atuam na ABS, bem como para a formação dos PEF que pretendem atuar neste contexto.
\end{abstract}

Palavras-chave: Sistema Único de Saúde; Atenção primária à saúde; Prática profissional; Estratégia Saúde da Família; Recursos humanos.

\section{ABSTRACT}

The objective of this essay was to present a proposal of the synthesis of the work of the Physical Education Professional (PEF) in Primary Health Care (PHC) in a brazilian context. We present the 15 items, elaborated from experiences lived in the field of actuation by the authors, as well as in the literature. Subsequently, the clarity and pertinence of the items were evaluated by 33 PEFs from nine different Brazilian municipalities that were currently working or who had previously worked in PHC. The items presented are not intended to compete or replace the guidelines of the Ministry of Health or the state or municipal secretariats. In fact, we hope that they may be useful for the PEF working in PHC, as well as for the professional qualification of the PEF who intend to work in this context.

Keywords: Unified Health System; Primary health care; Professional practice; Family Health Strategy; Human resources.

\section{(cc) BY-NC-SA}

Este obra está licenciado com uma Licença

\section{Introdução}

A partir, especialmente dos anos 2000, houve um aumento nas possibilidades de inserção do profissional de Educação Física (PEF) na Atenção Básica à Saúde $(\mathrm{ABS})^{1-3}$. Partindo-se do princípio que o PEF atuante na $\mathrm{ABS}$, em função das especificidades desta, não deve apenas repetir aquilo que faz em outros contextos, como academias e clubes, este ensaio teve como objetivo apresentar e discutir uma proposta de síntese para a atuação do PEF na ABS.

Importante destacar que este texto não busca competir com outras orientações, e mesmo diretrizes do
Ministério da Saúde e das secretarias estaduais e municipais, mas sim pretende trazer uma outra forma de síntese e, talvez, agregar novos elementos. Além disso, não deve ser interpretado como "livro de receitas", uma vez que reconhece-se que cada contexto tem suas próprias características e que mesmo as condições de trabalho de cada PEF são estruturantes no sentido de se ajudar a definir o que é ou não relevante e viável. Assim, é possível que em alguns contextos alguns itens apresentados não sejam adequados. Além disso, certamente outros itens poderiam ser apresentados, não tendo este trabalho o objetivo de "dar conta" de toda 
a complexidade da temática. Deste modo, cabe a cada PEF avaliar a pertinência e aplicabilidade de cada item em seu contexto de trabalho específico.

Vale mencionar que alguns dos itens que serão apresentados são semelhantes à algumas diretrizes de atuação profissional que aparecem nas diretrizes do Núcleo de Apoio à Saúde da Família (NASF) ${ }^{4}$, atualmente denominado Núcleo Ampliado de Saúde da Família e Atenção Básica (NASF-AB). No Cadernos de Atenção Básica ${ }^{4}$ em que são apresentadas as diretrizes do NASF, são apresentadas diretrizes para a atuação nas nove áreas estratégicas do NASF, sendo uma delas referente às "Práticas Corporais e Atividade Física". No entanto, é preciso destacar que nas diretrizes do NASF o foco é toda a equipe e não o PEF, como é o caso do presente texto. Além disso, mesmo sendo o NASF-AB a principal porta de entrada para os PEF atuarem na ABS, outras possibilidades existem, de modo que, acredita-se que este texto pode ser útil para PEF que atuam, por exemplo, no Programa Academia da Saúde, em programas estaduais ou municipais inseridos no contexto da ABS, nas residências multiprofissionais, entre outros.

\section{Considerações sobre a construção dos itens}

Inicialmente foram elaborados dez itens, a partir da experiência pessoal do primeiro autor deste trabalho, que foram apresentadas em algumas palestras e mesas redondas em diferentes estados brasileiros a partir de 2013. Muitas vezes, após estas falas, sugestões, críticas e comentários importantes foram realizados por diversas pessoas, especialmente PEF que atuavam no SUS. Assim, outros itens passaram a ser elaborados e incorporados, bem como alguns itens "originais" foram reelaborados.

Posteriormente, os itens foram revisados pelos outros dois autores deste trabalho, que realizaram acréscimos e modificações, chegando-se assim à 17 itens. De maneira adicional, alguns PEF que atuavam ou haviam atuado na $A B S$ por pelo menos seis meses foram convidados a avaliar a clareza e a pertinência de cada item. Esses PEF foram escolhidos por conveniência, a partir dos contatos que os autores tinham. Participaram 11 PEF de Florianópolis (SC), 10 de Londrina (PR), três de Recife (PE), dois de Lages (SC), dois de Rio Grande (RS), dois de São Bernardo do Campo (SP), um de João Pessoa (PB), um de Rio Claro (SP) e um de Paulista (PE). Após este feedback foram realizadas modificações, conforme sugestão dos PEF consultados. Destaca-se que esta consulta aos PEF que atuavam ou haviam atuado diretamente na ABS foi fundamental para a elaboração da versão final dos itens. Dois itens foram retirados, permanecendo, assim, 15 itens.

\section{Apresentação dos itens}

No quadro 1, os itens são apresentados, seguidos de apontamentos breves sobre cada um.

Quadro 1 - Síntese de itens a serem considerados na atuação do Profissional de Educação Física na Atenção Básica à Saúde.

\begin{tabular}{|l|}
\hline $\begin{array}{l}\text { Buscar atuação coerente com os princípios e diretrizes do Sistema Único } \\
\text { de Saúde }\end{array}$ \\
\hline Estar comprometido com a qualidade do serviço \\
\hline Buscar um bom conhecimento do território \\
\hline Participar do planejamento das ações de saúde da Atenção Básica à Saúde \\
\hline Buscar estimular e fomentar a participação e controle social \\
\hline $\begin{array}{l}\text { Buscar organizar e participar de atividades de educação em saúde, } \\
\text { considerando a cultura dos sujeitos e comunidades e buscando estabelecer } \\
\text { uma relação que respeite estes saberes, fugindo de uma concepção vertical } \\
\text { onde somente o profissional detém o saber }\end{array}$ \\
\hline Participar de atividades de apoio matricial \\
\hline $\begin{array}{l}\text { Reconhecer os múltiplos determinantes da atividade física (e de outros } \\
\text { comportamentos relacionados à saúde), evitando uma abordagem moralista } \\
\text { e que considere o estilo de vida apenas como consequência das escolhas } \\
\text { individuais de cada sujeito }\end{array}$ \\
\hline $\begin{array}{l}\text { Reconhecer a atividade física como objeto complexo e que a orientação } \\
\text { para a prática de atividade física não é exclusiva de uma única profissão }\end{array}$ \\
\hline Buscar fomentar a atuação intra e intersetorial para promoção da atividade física \\
\hline Não focar a atuação na prescrição individualizada \\
\hline $\begin{array}{l}\text { A organização de grupos para a prática de atividade física não deve ser a } \\
\text { única estratégia de atuação dos profissionais de EF }\end{array}$ \\
\hline $\begin{array}{l}\text { Na atuação junto aos grupos de prática de atividade física, buscar explorar a } \\
\text { riqueza de possibilidade das atividades físicas. }\end{array}$ \\
\hline Valorizar a utilização dos espaços públicos \\
\hline Buscar realizar avaliações permanentes \\
\hline
\end{tabular}

\section{1) Buscar atuação coerente com os princípios e dire-} trizes do SUS

Todo profissional inserido na ABS deve constantemente refletir a respeito da adequação de suas ações aos princípios finalísticos do Sistema Único de Saúde (universalidade, equidade e integralidade) ${ }^{5,6}$.

\section{2) Estar comprometido com a qualidade do serviço} Existe uma noção equivocada entre alguns profissionais envolvidos no SUS (e, igualmente lamentável, de parte da população, mídia, etc.) de que, como o SUS é um serviço público ele pode ser "qualquer coisa". Além disso, é preciso superar a noção de que o SUS é "coisa de pobre". Evidentemente que o SUS tem diversos problemas e desafios, mas também apresenta-se como uma importante possibilidade para a construção de uma sociedade melhor. 


\section{3) Buscar um bom conhecimento do território}

Assim como os demais membros da equipe, o PEF deve conhecer muito bem o território em que está inserido. Além das características das pessoas que estão no território, deve conhecer o que já acontece na comunidade, assim como a "história viva" do território. Saber aonde estão os espaços públicos de lazer, se existem outros programas de atividade física que acontecem na área, quais programas sociais existem, as escolinhas esportivas, as academias privadas e respectivas modalidades oferecidas, etc. Além disso, identificar as pessoas chave no processo de disseminação das informações necessárias para a maior abrangência das ações.

4) Participar do planejamento das ações de saúde da Atenção Básica à Saúde

O PEF não pode se achar, ou ser visto, como algo "complementar" à ABS. O PEF deve fazer parte efetivamente $\mathrm{da}(\mathrm{s})$ UBS em que está inserido. O PEF precisa participar deste planejamento, inclusive em função de seu potencial papel como articulador intra e intersetorial. Vale destacar que a promoção da atividade física é claramente um tema intersetorial, não sendo possível o setor saúde "dar conta" sozinho desta questão ${ }^{7}$.

\section{5) Buscar estimular e fomentar a participação e con- trole social}

A participação popular é fundamental para o desenvolvimento do SUS e a atuação do $\mathrm{PEF}$ pode ser rica neste sentido, inclusive estimulando, nos grupos específicos de prática de atividades físicas, que as pessoas da comunidade se organizem e participem de ações como o conselho local de saúde, associação de moradores, etc.

6) Buscar organizar e participar de atividades de educação em saúde, considerando a cultura dos sujeitos e comunidades e buscando estabelecer uma relação que respeite estes saberes, fugindo de uma concepção vertical onde somente o profissional detém o saber

As atividades de educação em saúde têm um grande potencial no sentido de promover conhecimentos e atitudes favoráveis à adoção de comportamentos saudáveis. Porém, faz-se necessário superar a lógica em que os profissionais de saúde são os únicos "detentores" do conhecimento e que cabe à comunidade uma participação meramente passiva neste processo. Alguns profissionais parecem limitar as ações de educação em saúde à "palestras". Evidentemente que em alguns momentos estas até podem ser importantes, mas certamente são, na maioria dos casos, insuficientes, e é possível que outras estratégias se façam necessárias e isso passa por estabelecer uma relação mais horizontal entre profissionais de saúde e comunidade. Além disso, estas ações devem ir além do simplesmente informar que atividade física é benéfica para a saúde, pois em geral isso já parece ser do conhecimento da maioria das pessoas e o desafio maior passa a ser o "como" fazer com que as pessoas sejam ativas fisicamente.

\section{7) Participar de atividades de apoio matricial}

As atividades de apoio matricial são fundamentais e, quando adequadamente realizadas, podem viabilizar um importante suporte técnico para as equipes e profissionais que irão atuar diretamente na atenção aos problemas de saúde ${ }^{8,9}$. Neste sentido, o compartilhamento de saberes se faz absolutamente necessário. Assim, o PEF deverá compartilhar muito de seus saberes e ao mesmo tempo estar aberto para aprender sobre outras áreas. $\mathrm{O}$ aconselhamento para atividade física pode ser uma importante estratégia para melhorar o matriciamento entre as equipes de saúde, ao capacitar a equipe de saúde para aconselhar, os PEF estão colocando em prática a lógica do apoio matricial.

8) Reconhecer os múltiplos determinantes da atividade física (e de outros comportamentos relacionados à saúde), evitando uma abordagem "moralista" que considere o estilo de vida apenas como consequência das escolhas individuais de cada sujeito Faz-se urgente que todos os PEF (e todos envolvidos com promoção de comportamentos saudáveis) superem a ideia de que o comportamento humano depende exclusivamente da vontade e das escolhas individuais de cada sujeito. Evidentemente a força de vontade e desejos individuais exercem influência, porém, não se pode ignorar outros determinantes, inclusive de ordem econômica, cultural, social, biológica, entre outros ${ }^{10}$. Ademais, os profissionais de saúde não existem para fazer julgamento moral do comportamento das pessoas, mas, sim, tem, entre outras funções, o papel de serem facilitadores no sentido de que a adoção de comportamentos considerados positivos para a saúde seja mais fácil por parte dos sujeitos e populações. 
9) Reconhecer a atividade física como objeto complexo e que a orientação para a prática de atividade física não é exclusiva de uma única profissão Assim como, por exemplo, a saúde mental é um tema central da Psicologia e a alimentação da $\mathrm{Nu}^{-}$ trição, a atividade física é da Educação Física. Porém, nenhum destes temas é exclusivo de uma única área do conhecimento, especialmente no contexto do SUS, onde o trabalho interprofissional deve ser constante, sendo necessário que todos os profissionais estejam muito abertos para aprender e para ensinar. Existem momentos em que o PEF deverá ensinar aspectos relativos à atividade física para outros membros da equipe, bem como em outros momentos deverá buscar aprender sobre temas diversos.

\section{0)Buscar fomentar a atuação intra e intersetorial para promoção da atividade física}

A característica complexa, multifatorial e multidimensional da atividade física exige a articulação com diferentes setores e com os distintos serviços e atores da rede de atenção em saúde. A ação do PEF tem potencial de ser importante nesta articulação.

\section{1)Não focar a atuação na prescrição individualizada} Assim como, por exemplo, um nutricionista não é contratado pelo SUS para prescrever dietas individualizadas (salvo alguns contextos mais específicos), o PEF também não é contratado para prescrever exercícios físicos desta maneira. Entretanto, nada impede que isso aconteça em alguns casos específicos. Por exemplo: um sujeito fisicamente ativo procura uma UBS aonde atua o PEF porque tem interesse em iniciar a prática de corrida de rua. Evidentemente não há nenhum problema em o profissional conversar com esta pessoa e, eventualmente, até prescrever exercícios individualmente para ela. Do mesmo modo, essa lógica também se aplica para indivíduos com condições clínicas muito específicas.

12)A organização de grupos para a prática de atividades físicas não deve ser a única estratégia de atuação dos PEF

Alguns PEF inseridos no SUS parecem limitar a sua atuação aos grupos de prática de atividades físicas ${ }^{11}$. Para isso, organizam grupos específicos (de caminhada, alongamento, de exercícios para diabéticos, hipertensos, etc.). Sem dúvida, esta pode ser uma estratégia importante. Talvez seja a mais óbvia e a com maior visibilidade, porém esta precisa estar articulada com outras. Ademais, a maior parte das pessoas que frequentam estes grupos apresentam características bem específicas (são, em geral, mulheres, de meia idade ou idosas, donas de casa, que já tem algum tipo de doença ou agravo crônico, que moram perto dos locais onde as práticas são realizadas, etc.) $)^{12}$ e as ações do PEF não podem ser somente para estas pessoas, mas, sim, precisam ser elaboradas para toda a comunidade do território de abrangência.

13) Na atuação junto aos grupos de prática de atividade física buscar explorar a riqueza de possibilidade das atividades físicas

Alguns profissionais parecem limitar seus programas ao tradicional: aquecimento/alongamento, seguido por algum tipo de exercício aeróbio (normalmente caminhada), depois algum exercício de resistência muscular localizada e finalizando com alguma atividade de relaxamento/volta a calma. Sugere-se que, na medida do possível, sejam inseridos outros elementos da cultura corporal, como dança, recreação, esportes, etc., atentando para a necessidade de adaptações à realidade e condição clínica dos participantes.

\section{4)Valorizar a utilização dos espaços públicos}

A ocupação do espaço público é de fundamental importância e pode inclusive ajudar a aumentar o senso de comunidade entre as pessoas. Estas precisam reconhecer o espaço público como um bem comum. Uma possível estratégia, é a alternância dos locais onde acontecem as práticas. Isto pode inclusive sensibilizar outras pessoas a participarem do grupo. Além disso, pode ser um elemento a mais de cobrança junto aos órgãos responsáveis para que a área tenha melhores condições para a prática de atividades físicas.

\section{5)Buscar realizar avaliações permanentes}

Sem dúvida os PEF devem ter alguma forma de avaliação de sua prática profissional. Evidentemente, a avaliação deve ser entendida de uma forma ampla, como parte importante de um processo e fundamental para proporcionar informações para uma melhor tomada de decisão. Ademais, destaca-se que estas avaliações não devem ser apenas focadas na aptidão física de pessoas que participam dos grupos formais. Sugere-se a inclusão de outras 
variáveis como por exemplo: comportamentos relacionados à saúde, participação social, qualidade de vida, atitudes, auto eficácia, conhecimento, etc. De qualquer modo, há de se ter cuidado com o excesso de medidas e testes e sempre considerar a coerência entre o que está sendo avaliado e os objetivos propostos. Avaliar por avaliar, além de representar uma importante perda de tempo, é desrespeitoso com as pessoas que se submetem à avaliação.

\section{Comentários finais}

Mais uma vez se faz necessário destacar que os itens apresentados neste ensaio não tem o objetivo de substituir orientações ou diretrizes do Ministério da Saúde ou outros órgãos competentes e que espera-se que este texto possa fornecer elementos para a reflexão de $\mathrm{PEF}$ inseridos na $\mathrm{ABS}$, de modo que estes possam avaliar, considerando as especificidades do seu contexto de atuação, a pertinência ou não de cada um dos itens apresentados.

Reafirma-se neste texto a necessidade de que o SUS se consolide enquanto Política de Estado e que possa ser de boa qualidade. Neste sentido, acredita-se que a atividade física pode ter um papel importante. Não só devido à sua relação com as doenças e agravos crônicos de saúde, mas também por seu aspecto cultural e sua potência no sentido de contribuir para a mudança do modelo de atenção, privilegiando um olhar ampliado de saúde, coerente com o princípio da integralidade inclusive, em detrimento ao olhar biomédico, ainda hegemônico.

\section{Conflito de interesses}

Os autores declaram não haver conflito de interesses.

\section{Contribuição dos autores}

Loch MR, concebeu a ideia original do manuscrito, elaborou a versão inicial dos itens, do manuscrito e participou de todas as etapas da construção do manuscrito. Dias DF e Rech CR, realizaram análise crítica da versão inicial do manuscrito, contribuíram na formulação de novos itens e na revisão dos itens, bem como da versão final do manuscrito.

\section{Referências}

1. Santos $S$, Benedetti TRB. Cenário de implantação do Núcleo de Apoio a Saúde da Família e a inserção do profissional de Educação Física. Rev Bras Ativ Fís Saúde. 2013;17(3):188-94.

2. Rodrigues JD, Ferreira D, Silva P, Caminha I, de Farias Junior JC. Inserção e atuação do profissional de educação física na atenção básica à saúde: revisão sistemática. Rev Bras Ativ Fís Saúde. 2013;18(1):05-15.

3. Loch MR, Knuth AG, Silva ICM, Guerra PH. As práticas corporais/atividade física nos 30 anos do Sistema Único de Saúde. Ciênc Saúde Colet. 2018; 23(10):3469.

4. Brasil. Ministério da Saúde. Secretaria de Atenção à Saúde. Departamento de Atenção Básica. Cadernos de Atenção Básica n. 27 - Diretrizes do NASF - Núcleo de Apoio à Saúde da Família. Brasília; 2009. Disponível em http:// bvsms.saude.gov.br/bvs/publicacoes/caderno_atencao_ basica_diretrizes_nasf.pdf

5. Paim J. O que é o SUS. Rio de Janeiro: Editora FIOCRUZ; 2009.

6. Paim J, Travassos C, Almeida C, Bahia L, Macinko J. The Brazilian health system: history, advances, and challenges. Lancet. 2011;377(9779):1778-97.

7. Reis RS, Salvo D, Ogilvie D, Lambert EV, Goenka S, Brownson RC. Scaling up physical activity interventions worldwide: stepping up to larger and smarter approaches to get people moving. Lancet. 2016;388(10051):1337-48.

8. Campos GWS, Domitti AC. Apoio matricial e equipe de referência: uma metodologia para gestão do trabalho interdisciplinar em saúde. Cad Saúde Pública. 2007;23(2):399-407.

9. Barros JO, Gonçalves RMA, Kaltner RP, Lancman S. Estratégia do apoio matricial: a experiência de duas equipes do Núcleo de a poio à saúde da família (NASF) da cidade de São Paulo, Brasil. Ciênc Saúde Colet. 2015;20(9):2847-56.

10. Bauman AE, Reis RS, Sallis JF, Wells JC, Loos RJ, Martin BW, et al. Correlates of physical activity: why are some people physically active and others not? Lancet. 2012;380(9838):258-71.

11. Souza SC, Loch MR. Intervenção do profissional de Educação Física nos Núcleos de Apoio à Saúde da Família em municípios do norte do Paraná. Rev Bras Ativ Fís e Saúde. 2011;16(1):5-10.

12. Loch MR, Rodrigues CG, Teixeira DC. E os homens? E os que moram longe? E os mais jovens?...? Perfil dos usuários de programas de atividade física oferecidos pelas Unidades Básicas de Saúde de Londrina-PR. Rev Bras Ciêc Esporte. 2013;35(4):947-61.

Recebido: 26/09/2018

Aprovado: 10/07/2019

Como citar este artigo:

Loch MR, Dias DF, Rech CR. Apontamentos para a atuação do Profissional de Educação Física na Atenção Básica à Saúde: um ensaio. Rev Bras Ativ Saúde.2019;24:e0069. DOI: 10.12820/rbafs.24e0069 